\title{
SOBRE LA VALIDEZ DE PACHYCHILA FREYI KOCH, 1944, P. DENTICOLLIS GRIMM, 1987 Y LAS SINONIMIAS DEL GÉNERO INTRODUCIDAS POR VIÑOLAS \& CARTAGENA (2005) EN SU FAUNA DE TENEBRIÓNIDOS (ARGANIA EDITIO) (COLEOPTERA, TENEBRIONIDAE, PIMELIINAE)
}

\author{
J. Ferrer ${ }^{1}$, J. L. Bujalance ${ }^{2}$ y A. Castro Tovar ${ }^{3}$
}

\begin{abstract}
RESUMEN
Pachychila freyi Koch, 1944 -confundida con Pachychila incrassata Rosenhauer, 1856-, y P. denticollis Grimm, 1987, son dos especies válidas de Almería, España, arbitrariamente puestas en sinonimia de Pachychila glabella (Herbst, 1799) y de P. germari Solier, 1835, respectivamente, por Viñolas \& Cartagena (2005). Ambas son claramente diferentes de estas especies, y de todas las Pachychilas conocidas y descritas, por un gran número de caracteres morfológicos, ignorados por estos autores, que no han examinado los tipos. Además, "Pachychila germari Solier" (Viñolas \& Cartagena, 2005), presenta considerables diferencias en su genitalia con Pachychila germari del norte de África, y en realidad se trata de Pachychila bifida Rosenhauer,1856, otra especie válida de la fauna ibérica.
\end{abstract}

Palabras clave: Península Ibérica, Almería, Coleoptera, Tenebrionidae, Pimeliinae, Tentyriini, Pachychila bona species, no sinonimia.

\begin{abstract}
About the validity of Pachychila freyi Koch, 1944, P. denticollis Grimm, 1987 and the synonymies proposed by Viñolas \& Cartagena (2005) in Fauna de Tenebriónidos (Argania Editio) (Coleoptera, Tenebrionidae, Pimeliinae)

Pachychila freyi Koch, 1944, misidentified as Pachychila incrassata Rosenhauer, 1856 by Viñolas \& Cartagena (2005), and P. denticollis Grimm, 1987 are two valid species from Almería, Spain, arbitrarily treated as junior synonyms respectively of Pachychila glabella (Herbst, 1799) and of P. germari Solier, 1835. They differ clearly from Pachychila glabella and P. germari and from all other described species of this genus, by several morphological external and internal characters, ignored by the authors, who failed to study types. Moreover, the insect treated as "Pachychila germari Solier" by Viñolas \& Cartagena (2005), exhibits significant genitalia differences compared to Pachychila germari from North Africa, and in reality belongs to Pachychila bifida Rosenhauer, 1856, another valid species of the Iberian fauna.

Key words: Iberian Peninsula, Almeria, Coleoptera, Tenebrionidae, Pimeliinae,
\end{abstract} Tentyriini, Pachychila bona species, non synonymy.

Swedish Museum of Natural History, Department of Entomology, S-10405 Stockholm, Sweden

Plaza de Blas Infante 1, E-14850 Baena, España.

Travesía General Castaños n. 2, $2^{\circ}$ piso CP. 23001 Jaén, España. 


\section{Introducción}

El objeto de esta nota es continuar corrigiendo un cierto número de errores taxonómicos que afectan la representación ibero-balear de la fauna de Coleópteros Tenebriónidos, tal como han sido presentados en una obra reciente de Argania Editio por Viñolas \& Cartagena (2005). Errores que son sobre todo resultado de sobreestimar la validez de determinaciones previas de las colecciones examinadas, sin verificación del material típico (Ferrer, 2006), un cierto desconocimiento de la actual sistemática del grupo (Bouchard, 2006), unido a un aislamiento voluntario (Cárdenas et al., 2006).

\section{Material y Método}

Para determinar correctamente nuestro material, JLB obtuvo en préstamo ejemplares del género Pachychila del Museo Nacional de Ciencias Naturales (MNCN) de Madrid, especialmente material determinado por Pérez Arcas. ACT prospectó intensamente las zonas de la provincia de Almería que constituyen el hábitat de los taxones estudiados y JF se desplazó a París para estudiar los tipos de Solier y de Rosenhauer, depositados en el Muséum National d'Histoire Naturelle (MNHN), así como la colección de Peyerimhoff, donde se examinaron también ejemplares del género Pachychila determinados por todos los autores que se han ocupado de él, así como material determinado in litt. por P. Ardoin, F. Español y A. Cobos.

Asimismo, obtuvimos en préstamo los tipos de los Tentyriini ibéricos descritos por Carlo Koch, conservados en la colección G. Frey, del Naturhistorisches Museum, Basel, y de Pachychila denticollis Grimm, 1987, de nuestro colega, el Dr. Roland Grimm de Tübingen. Este material típico fue confrontado con el material de Francisco Español obtenido hace tiempo por JF, depositado en la colección J. Ferrer, así como con el del
Naturhistoriska riksmuseet, Estocolmo, donde se conservan especímenes de Pachychila estudiados por A. Schuster $(1919,1920)$ para sus revisiones del género. En fin, para no deteriorar los delicados ejemplares tipo de estas especies de Pachychila, se seleccionaron especímenes semejantes a los tipos respectivos para extraer y examinar la genitalia.

\section{Breve historia del género}

La primera especie de Pachychila fue descrita por Linnaeus en 1758, en el Systema Naturae, bajo el nombre de Tenebrio caraboides, nomen oblitum de Pachychila hispanica Solier, 1835, cuyos sintipos se encuentran en el Museo de la Evolución de Uppsala y en el Museo de París (coll. Marseul, MNHN), y son objeto de un estudio en preparación.

Herbst (1779) describe y representa por primera vez una Pachychila, bajo el nombre de Pimelia glabella, que constituye una especie bien conocida, gracias a la figura que acompaña la descripción original.

El género Pachychila fue creado por Escholschz en 1831, para recibir una especie descrita en esa ocasión, P. subovata de Sicilia, así como Pimelia punctata Fabricius, 1798. Solier realizó en 1835 la primera revisión indicando exhaustivamente sus caracteres, que fueron adoptados seguidamente por todos los autores. El nombre del género fue indebidamente enmendado a Pachychile por Lacordaire en 1859. Las especies andaluzas fueron estudiadas por Rosenhauer (1856), el conjunto de su representación por Reitter (1900), así como por Schuster (1919, 1921), el cual revisó los géneros Pachychilina Reitter, 1900 y Pachychila, seguido de Peyerimhoff (1927), quien estudió especialmente la representación norteafricana del género, representando por primera vez los edeagos. Gebien (1910, 1937) realizó el catálogo del género y Antoine (1942) estudió detalladamente las especies marro-

Figs. 1-20.- 1) Epistoma, puntuación y antenas de Pachychila freyi, 2) P. denticollis, 3) P. bifida, 4) P. glabella y 5) P. germari. 6) Mentum de Pachychila freyi, 7) P. germari, 8) P. denticollis, 9) P. glabella y 10) P. bifida. 11) Reborde basal del pronoto de Pachychila glabella, 12) P. freyi, 13) P. denticollis, 14) P. bifida y 15) P. germari. 16) Esternito anal de Pachychila denticollis, 17) P. bifida, 18) P. freyi, 19) P. germari y 20) P. glabella.

Figs. 1-20.-1) Epistome, punctuation and antennas of Pachychila freyi, 2) P. denticollis, 3) P. bifida, 4) P. glabella and 5) P. germari. 6) Mentum of Pachychila freyi, 7) P. germari, 8) P. denticollis, 9) P. glabella and 10) P. bifida. 11) Basal margin of pronotum of Pachychila glabella, 12) P. freyi, 13) P. denticollis, 14) P. bifida and 15) P. germari. 16) Anal sternite of Pachychila denticollis, 17) P. bifida, 18) P. freyi, 19) P. germari and 20) P. glabella. 


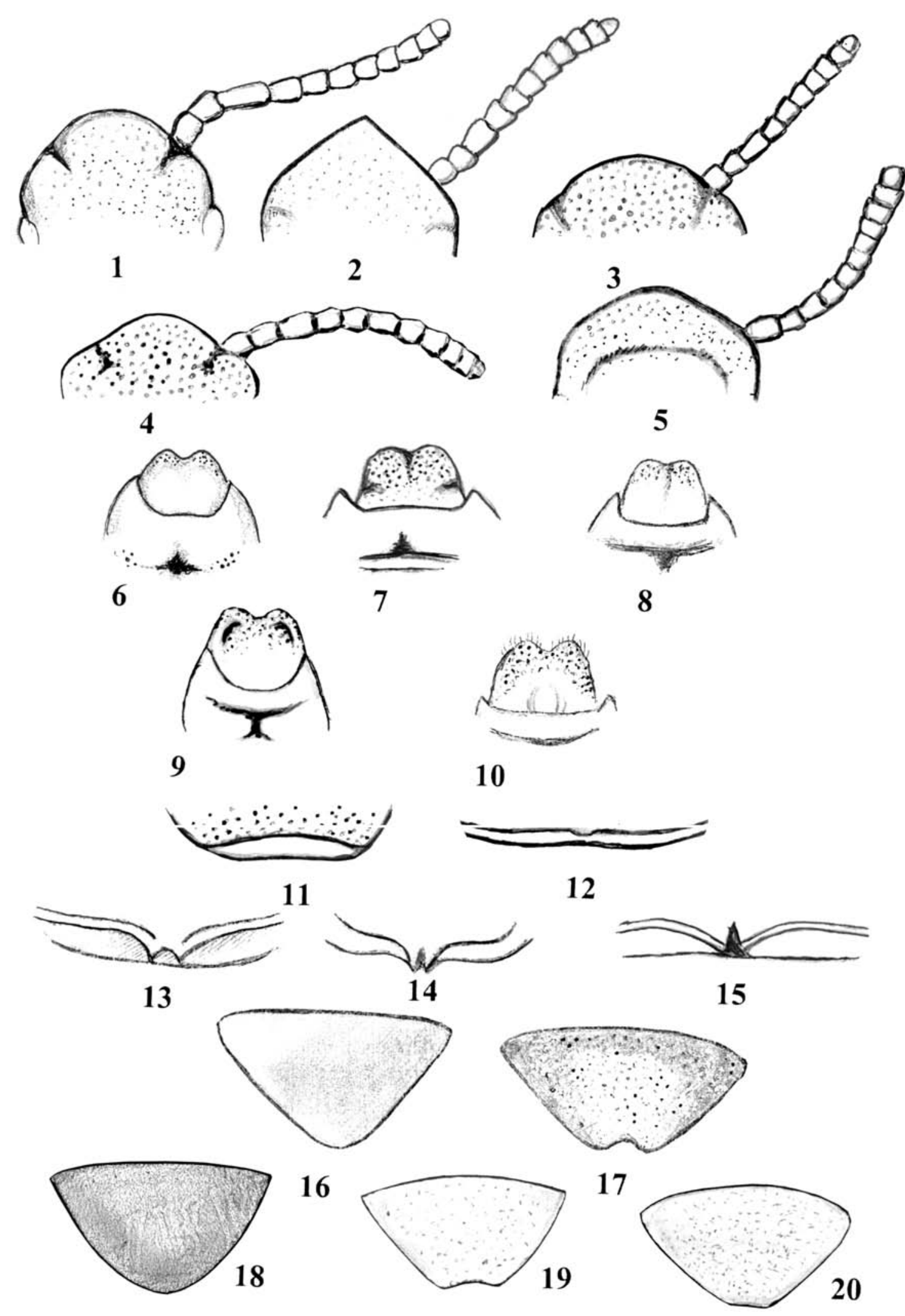


quíes mientras que Koch (1944) se refirió sobre todo a las andaluzas y describió una especie nueva, P. freyi. Español (1944) estudió las Pachychila en España y Baleares y Grimm (1987) ilustró algunas especies andaluzas y describió Pachychila denticollis. En fin, Viñolas \& Cartagena (2005) han tratado de realizar una síntesis que no se ajusta al material típico examinado por los autores de esta nota, ya que, entre otros errores, Pachychila freyi y $P$. denticollis son indebidamente puestas en la sinonimia de Pachychila glabella (Herbst, 1799) y de $P$. germari Solier, 1835, respectivamente, sin ningún examen de los tipos. La validez de estas especies no deja ningún lugar a dudas estudiando las descripciónes originales y las figuras proporcionadas por Grimm (1987), ya que tanto Pachychila freyi como $P$. denticollis ocupan, a juzgar por su descripción y el detallado estudio que hace este autor, un lugar perfectamente definido en el género por el conjunto de sus caracteres.

\section{Especies estudiadas}

Pachychila germari Solier, 1835 (figs. 5, 7, 15, 19, 27-30, 37)

MATERIAL TÍPICO EXAMINADO: Tipo y dos sintipos: germari/Sol. T/ Alger Dre 64/coll. Solier coll. Marseul (MNHN, París).

Material adicional eXAminAdo: Argelia: Orán, Bona (coll. Peyerimhoff) (MNHN); Marruecos: playa Río Smir, VIII.1975, Ferrer Andreu leg.; Túnez: Tunisie, Sousse (coll. Vauloger) (MNHN), Sousse (Coll. J. Ferrer).

DiAGNOSIS: De color negro brillante, con el epistoma redondeado y engrosado en su borde anterior, caracterizada por el pronoto netamente transverso y el saliente bilobulado (en vez de bífido) del borde basal del pronoto, con dos dientecillos bien separados (más largos y más juntos en bifida).

Pachychila germari es una especie norteafricana cuya área de distribución es desconocida, por estar hasta el momento confundida con Pachychila bifida, propia de la Península Ibérica, y también con las Pachychila, asimiladas a "germari" de Cerdeña, Sicilia y de Túnez, pendientes de revisión.
Pachychila bifida Rosenhauer, 1856

[bona species non sinonimia de $P$. germari Solier, 1835]

(figs. 3, 10, 14, 17, 22, 23, 31, 32, 38)

Material tíPICO EXAMINADO: Holotipo: Pachychila germari bifida/Type/ Espagne Deyr 54/coll. Solier/coll. Marseul 184220. MNHN; París.

Material adicional EXAminado: Numerosos ejemplares de Estepona, provincia de Málaga (Swedish Museum of Natural History, Estocolmo); de Algeciras, provincia de Cádiz, Pachychila germari Solier, F. Español det. (coll. J. Ferrer), NRM, Estocolmo; San Roque, VIII.1975, Ferrer Andreu leg.

Diagnosis: Especie de Andalucía, bien diferente de la verdadera Pachychila germari, que representa, a juzgar por la genitalia de ambos sexos, una superespecie formada por la forma típica de Argelia, cuyo ovipositor y edeago (figs. 27-28) son completamente diferentes del de los taxones indebidamente sinonimizados con germari por Peyerimhoff (1927) a título de variedades; criterio seguido por Español (1944) y por Viñolas \& Cartagena (2005), que tampoco examinaron los edeagos, ni mucho menos los ovipositores (figs. 22-23). Sus hábitus son muy diferentes (fotos $37 \mathrm{cf} 38$ ).

\section{Pachychila denticollis Grimm, 1987 [bona species, non sinonimia de $P$. germari Solier, 1835]} (figs. 2, 8, 13, 16, 21, 24, 40)

Material tíPICO EXAMINADO: Holotipo: macho, Südspanien, prov. Almería, Cabo de Gata, 4 km SW San José, 26.9.1984, leg. Grimm and Rachinski; in coll. R. Grimm, Tübingen.

Paratipo: hembra, "Südspanien, prov. Almeria, Cabo de Gata, 4 km SW San José, 26.9.1984, leg. Grimm and Rachinski; in coll. Grimm, Tübingen.

Material adicional eXaminado: Almería, Playa de Monsul, San José, 14.XII.2004, A. Castro Tovar leg. (1 macho).

DiAgnosis: Pachychila denticollis se reconoce a primera vista y se diferencia fácilmente de bifida, por la combinación del conjunto de caracteres exhaustivamente descritos por Grimm, haciendo énfasis en la genitalia, ovipositor (fig. 21) y edeago (fig. 24), los cuales son completamente diferentes. También se distingue por sus antenas, proporcionalmente mucho más gruesas y tan largas como la anchura cefálica -las de bifida ("germari") más cortas y las de denticollis

Figs. 21-32.-21) Ovipositor de Pachychila denticollis, 22) P. bifida y 28) P. germari. 23) Edeago de P. bifida, 24) P. denticollis, 25) P. freyi, 26) P. glabella y 27) P. germari. 29-30) Protibia de $P$. germari y 31-32) P. bifida.

Figs. 21-32.-21) Ovipositor of. Pachychila denticollis, 22) P. bifida and 28) P. germari. Aedeagus of P. bifida, 24) P. denticollis, 25) P. freyi, 26) P. glabella and 27) P. germari. 29-30) Protibia of P. germari and 31-32) P. bifida. 


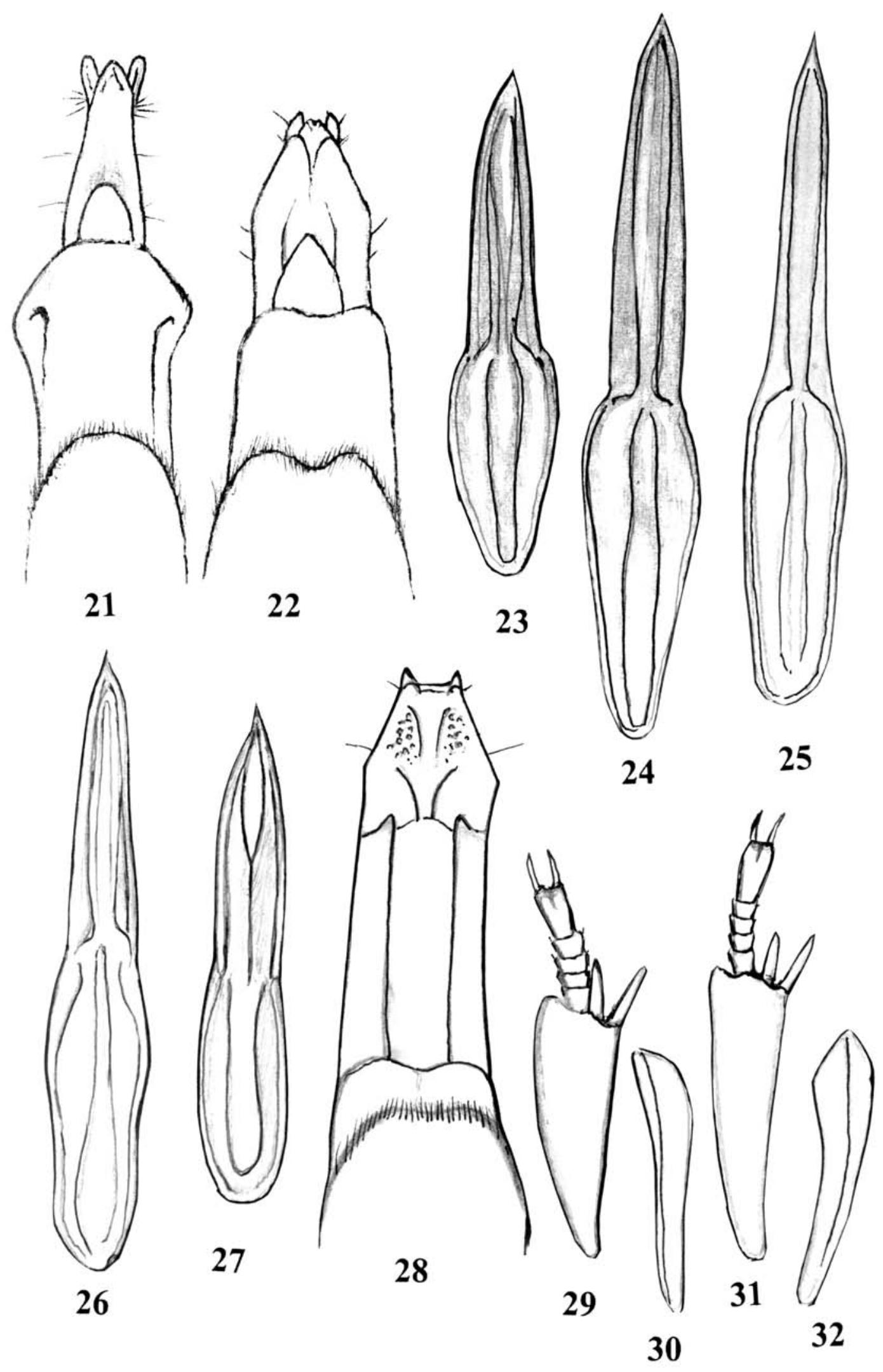



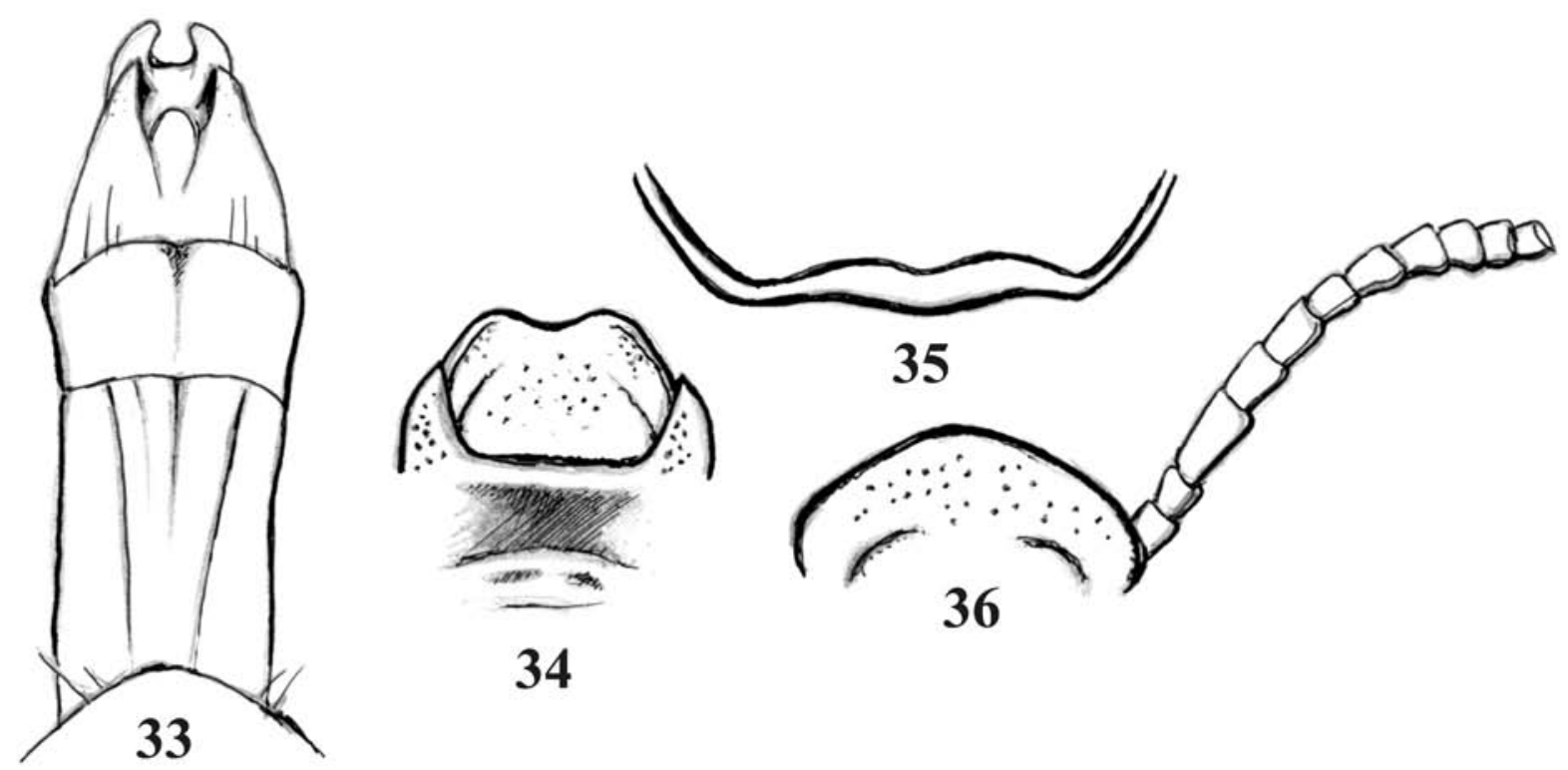

Figs. 33-36.- Pachychila incrassata: 33) Ovipositor, 34) mentum, 35) borde basal del pronoto y 36) borde anterior de la cabeza.

Figs. 33-36.- Pachychila incrassata: 33) Ovipositor, 34) mentum, 35) basal margin of pronotum and 36) anterior board of head.

mucho más largas-, por la forma triangular (no redondeada) del epistoma y por la puntuación mucho más grosera de bifida (fig. 2 cf. 3); por la forma, el punteado y la pubescencia totalmente diferente del mentum y del surco gular (fig. 8); porque denticollis presenta una incisión fosular que no existe en bifida (fig. 10); por la diferente escotadura basal del pronoto (figs. 1314); por tener el macho de denticollis el esternito anal perfectamente redondeado en el ápice (fig. 16) y carecer de la muesca o entalladura apical, característica de bifida (fig. 17) y de germari (fig. 19), presentando un aspecto completamente liso y mate, mientras que está fina y densamente punteado en bifida; por su talla mayor, alcanza hasta $11 \mathrm{~mm}$; por el aspecto mate de la superficie dorsal, debido a una escultura de la cutícula diferente, formada por mallas isodiamétricas microscópicas distintas; y por presentar las protibias más alargadas y macizas (figs. 31-32).

\footnotetext{
Pachychila freyi Koch, 1944

[bona species, non sinonimia de Pachychila glabella (Herbst, 1799)]

(figs. 1, 6, 12, 18, 25, 41)

= Pachychila incrassata Viñolas \& Cartagena, 2005: 353, foto d, non Pachychila incrassata Rosenhauer, 1856
}

MAterial tíPICO EXAMINAdo: Holotipo: Almería, Roquetta, Hisp. m. V.1943 leg. Frey, C. Koch, in den litoralen Dünen unter Pflanzenbüschen, in sand vergraben (Naturhistorisches Museum, Basel).

Material adicional eXAminado: Almería, La Garrofa, 15.III.1950, Cobos Sánchez leg./ Pachychila freyi Koch det. A. Cobos (MNHN); Garrocha, 24.VII.1959, A. Fages leg./P. Ardoin (MNHN).

Diagnosis: Especie simpátrica de Pachychila denticollis, sin posible confusión con ninguna otra Pachychila por la combinación de sus caracteres exhaustivamente expuestos por Koch (1944) e ilustrados por Grimm (1987), fácilmente reconocible por su epistoma redondeado, las antenas largas, la puntuación pronotal mucho más fina que en glabella, el reborde basal del pronoto subparalelo a la base de los élitros, bastante más fino y mucho más estrecho que el de glabella (fig. 11) y sobre todo mucho más alargado (fig. 12), por su edeago de parámeros alargados y más estrecho, aguzado en el ápice (fig. 25), muy distinto del de glabella (fig. 26) que presenta la base parameral ancha y sinuada. El holotipo de Pachychila freyi (fig. 41) es, por otra parte, idéntico a la fotografía de Pachychila que Viñolas \& Cartagena (2005: 353 d) utilizan para representar Pachychila incrassata Rosenhauer. 

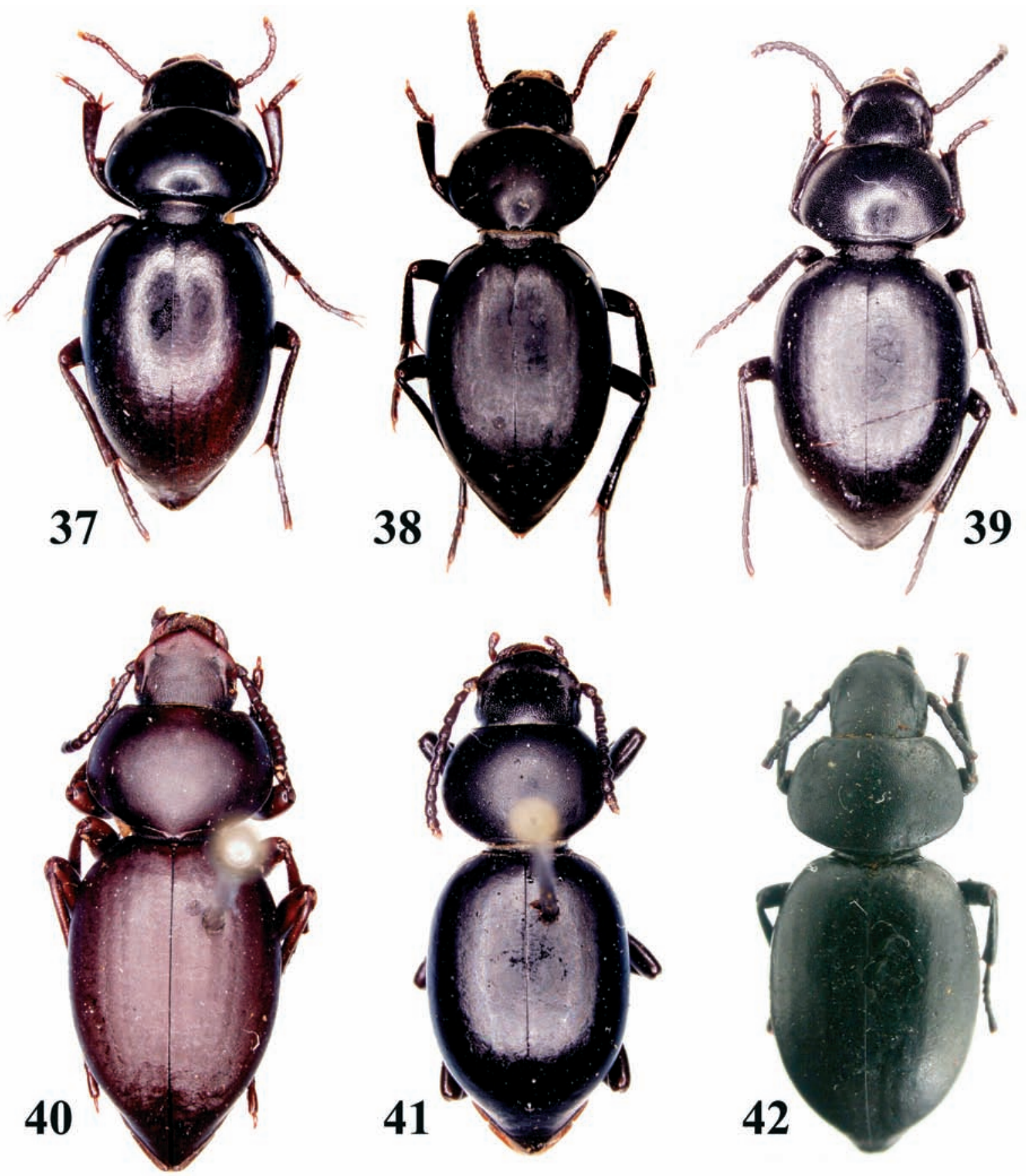

Figs. 37-42.-37) Pachychila germari de Alger, Argelia. 38) Pachychila bifida de Estepona, Málaga. 39) Pachychila glabella de Toledo. 40) Holotipo de Pachychila denticollis. 41) Holotipo de Pachychila freyi. 42) Pachychila incrassata de Loja, Granada.

Figs. 37-42.- 37) Pachychila germari from Alger, Algeria. 38) Pachychila bifida from Estepona, Málaga. 39) Pachychila glabella from Toledo. 40) Holotype of Pachychila denticollis. 41) Holotype of Pachychila freyi. 42) Pachychila incrassata from Loja, Granada. 
Pachychila incrassata Rosenhauer, 1856 (non Pachychila incrassata Viñolas y Cartagena, 2005: 353, foto d)

(figs. 33, 34, 35, 36, 42)

MATERIAL TíPICO EXAMINADO: Holotipo: Pachychila incrassata type de Rosenhauer/ 224 coll. L. Bedel/ coll. Marseul (MNHN). Material adicional eXAminado: Pachychila incrassata Rsh /Pérez Arcas det. Loja (MNHN); Sierra de Alfacar, Granada/ incrassata A. Schuster det. (NRM).

Diagnosis: Especie inconfundible, muy distinta de todas las especies citadas por el aspecto rechoncho, el pronoto fuertemente transverso, el doble de ancho que de largo, con el reborde basal alargado y muy sinuoso, de lados ampliamente redondeados, con los élitros proporcionalmente más cortos que en $P$. glabella.

\section{Discusión}

La clave presentada por estos autores para clasificar las especies ibéricas de Pachychila no permite la clasificacíon de Pachychila denticollis (ni tampoco de Pachychila incrassata).

Veamos si es una clave deficiente o, si por el contrario, la clave en principio funciona, pero no permite la determinación de esta especie, indicando su validez específica. El comienzo de la clave reza como sigue:

1 Borde basal del pronoto engrosado o ensanchado en una amplia zona central (Fig. 100)

- Borde basal del pronoto igual y delgado en toda su longitud o salvo en algunos casos con un estrecho saliente en la zona central (fig. 101 y 102)

Pachychila denticollis no entra en esta claves por tener el borde basal del pronoto engrosado en toda su longitud (fig. 13), carácter combinado con una foseta subgular (fig. 8). La primera opción llevaría por tanto esta especie al apartado 2, y de éste al apartado 5, a causa de su foseta gular; en el apartado 5, su puntuación cefálica la situaría entre incrassata y glabella, precisamente como anuncia Grimm en su diagnosis. La segunda opción -a todas luces errónea, ya que su pronoto no puede considerarse finamente rebordeado o sin rebordellevaría a esta especie al apartado 8, donde sitúan "germari". Pero en este apartado no puede tampoco clasificarse, pues su Pachychila "germari", es decir bifida, tiene el epistoma "truncado", o sea, levemente redondeado (fig. 3) pero no subdenticular, como P. denticollis (fig. 2). Su epistoma netamente triangular la situaría hipotéticamente junto a frioli. Y en ese caso la sinonimia con germari sería asimismo falsa.

\section{Resultados}

El examen del material típico de las citadas especies de Pachychila, descritas por Solier y Rosenhauer, conservado en el Muséum National d'Histoire Naturelle, París, confirma que las dos especies recientemente descritas por nuestros colegas alemanes no pueden ponerse en sinonimia, como hacen Viñolas \& Cartagena (2005), con ninguna Pachychila descrita anteriormente (Solier, 1835; Rosenhauer, 1856; Reitter, 1900; Schuster, 1919, 1921; Peyerimhoff, 1927; Antoine, 1942; Español, 1944; Koch, 1944), tanto por los caracteres descritos por sus autores respectivos, como por otros que damos a conocer y que soportan claramente su identidad específica. Asimismo, en la ordenación del género que presentan se confunden otros taxones, como la verdadera Pachychila incrassata Rosenhauer, y además se ignoran los límites geográficos de Pachychila bifida Rosenhauer, confundida a su vez con Pachychila germari. Por todo ello recomendamos que este trabajo sea manejado con suma circunspección, dado el número de errores que contiene.

\section{AGRADECIMIENTOS}

Al Dr. Claude Girard, Laboratoire d'Entomologie, del Muséum national d'Histoire naturelle, París; a la Dra. Carolina Martín, del Museo Nacional de Ciencias Naturales, Madrid; y a la Dra. Eva Sprecher, del Naturhistorisches Museum, Basel, por permitirnos el estudio de los Tentyriini descritos por Carlo Koch, depositados bajo su custodia. Al Dr. Roland Grimm, Tübingen, por su cortesía al prestarnos los tipos de Pachychila denticollis, al Dr. Kjell Arne Johanson, Bert Gustafsson, Bert Viklund y Niklas Jönsson del Swedish Museum of Natural History, Estocolmo, y al Dr. Hans Mejlon, del Museo de la Evolución de la Universidad de Uppsala, por su caluroso apoyo en nuestro trabajo.

\section{Referencias}

Antorne, M., 1942. Notes d'entomologie marocaine, XXXVI. Observations sur les Pachychila (Col. Tenebrionidae). Bulletin de la Société de Sciences naturelles du Maroc, 22: 59-82.

Bouchard, P., 2006. Analyse of Books. Fauna de Tenebriónidos de la Península Ibérica y Baleares, Viñolas, A. y Cartagena, M.C. Argania Editio, vol. 1. Lagriinae y Pimeliinae, $428 \mathrm{pp}$. Bulletin of the Entomological Society of Canada, 38(2): 66-67. 
Cárdenas, A. M., Bujalance, J. L. \& Hidalgo, J. M., 2006. Sobre la presencia de Stenosis mogadorica (Col. Tenebrionidae) en Europa: replica a los comentarios de VIÑOLAS y CARTAGENA (2005) en relación a esta especie. Boletín de la Sociedad Entomológica Aragonesa, 39: 415-416.

Eschscholtz, J. F., 1831. Zoological Atlas enthaltend Abbildungen neuer Thieralten während Kotzebue's zweiter Reise um die Welt 1823-26 gesammelt, Heft 5. Reimer. Berlin. 184 pp. + 4 Taf.

ESPAÑOL, F., 1944. Datos para el conocimiento de los Tenebriónidos del Mediterráneo occidental. 5. El género Pachychila en España y Baleares. Graellsia, 2(4): 101-108.

FERrer, J., 2006. Revisión crítica del libro: Fauna de Tenebriónidos de la Península Ibérica y Baleares, vol. 1. Lagriinae y Pimeliinae, Viñolas \& Cartagena, 2005 y propuesta de nueva sinonimia. Boletín de la Sociedad Entomológica Aragonesa, 39: 463-466.

Gebien, H., 1910. Katalog der Tenebrioniden, pars 15, 1. W. Junk und S. Schenkling. Berlin. 252 pp.

Gebien, H., 1937. Katalog der Tenebrioniden. Teil 1. Tip. D. del Bianco. Udine. 381 pp.

Grimm, R., 1987. Pacychila denticollis sp. n. aus Südspanien (Coleoptera, Tenebrionidae). Nachrichtenblatt der Bayerischen Entomologen, 36(1): 21-24.

Herbst, J. F. W., 1799. En: C. G. Jablonski, Natursystem aller bekannten in- und ausländsischen Insekten als eine Fortsetzung der von Büffonschen Naturgeschichte. Der Käfer, bd. 8. J. Pauli. Berlin. 420 pp.

$\mathrm{KocH}, \mathrm{C}$, 1944. Tenebrionidae I. Beitrag zur Kenntnis der Iberischen Faunan. Mitteilungen der Münchner Entomologisches Gesellschaft, 5(34): 215-254.

LACORDAIRE, T. H., 1859. Histoire Naturelle des Insectes. Genera des Coléoptères, Tome 5(2). Roret. Paris. pp. 401-750 pp.

Linnaeus, C. von, 1758. Caroli Linnaei Systema Naturae - A photographic facsimile of the first Volume of the Tenth edidion (1758) Regnum Animale. Trustees of the British Museum of Natural History. London. 1956. 824 pp.
Peyerimhoff, P., 1927. La systématique des Coléoptères du Nord Africain. 1 Les Pachychila. Tenebrionidae. L'Abeille, 34: 1-54.

ReitTer, E., 1900. Bestimmungstabelle der Tenebrioniden.Abteilungen: Tentyriini und Adelostomini aus Europa und der angrenzenden Ländern. Verhandlungen des Naturforschenden Vereins in Brunn, 39: 8297.

Rosenhauer, W. G., 1856. Die Thiere Andalusiens nach den Resultaten einer reise zusammengestellt nebst den Beschreibungen von 249 neuen oder bis zetzt noch unbescreibenen Gattungen un Arten. Erlangen. Blaesing. 429 pp., 3 taf.

Schuster, A., 1919. Revision der TenebrionidenGattungen Pachychilina Reitter und Pachychile Eschscholtz. Koleopterologische Rundschau, 8: 2743.

Schuster, A., 1921. Revision der TenebrionidenGattungen Pachychilina Reitter und Pachychile Eschscholtz. Koleopterologische Rundschau, 9: 1- 27.

Solier, J. A. A., 1935. Essai sur les Coléoptérides (Suite) Tentyriites,2. Annales de la Société entomologique de France, 4: 249-419.

Viñolas, A. \& Cartagena, M. C., 2005. Fauna de Tenebriónidos de la Península Ibérica y Baleares, vol. 1. Lagriinae y Pimeliinae. Argania Editio. Barcelona. 428 pp
Recibido, 31-X-2006 Aceptado, 29-III-2007 Publicado, 27-VI-2007 